\title{
Nietzsches Anthropologie des produktiven Antagonismus
}

Dass der Mensch in sich selbst und im Verhältnis zu den Anderen ein Zusammenhang gegensätzlicher Kräfte ist, kann als ein zentraler Gedanke Nietzsches gelten. Das Element des Gegensätzlichen kommt im Titel meiner Ausführungen unter dem Stichwort ,Antagonismus‘ zum Ausdruck. Nietzsche benutzt diesen Begriff in interessanter und einschlägiger Weise, wie wir sehen werden. Geläufig ist der Terminus aber nicht zuletzt aus dem Umkreis der Anthropologie Kants. Hier bezeichnet er eine grundlegende Spannung, die in der „menschlichen Natur“ angelegt sei und die Kant ,gesellige Ungeselligkeit“ nennt (Kant 1912, 20). Der Tendenz, sich zu vergesellschaften und in ein soziales Gefüge einzuordnen, steht die Tendenz, sich zu vereinzeln und zu isolieren, entgegen. Der Mensch ist gesellig, weil er sich nur als einer unter anderen entwickeln und in jeder nur denkbaren Hinsicht zum Menschen bilden kann. Zugleich ist er ungesellig, weil er alles nach seinem eigenen „Sinne“ aus- und einrichten möchte. Insofern ist der Mensch, wie Kant schreibt, zum „Widerstand“ gegen Andere geneigt (ebd. 21). Von diesen freilich wird er im Gegenzug ebensolchen Widerstand gegen sich und seine von „Ehrsucht, Herrschsucht oder Habsucht“ getriebenen Ambitionen erwarten (ebd.). Denn auch sie besitzen den beschriebenen Eigensinn. Und doch können die so kapriziös gegeneinander eingestellten und gegeneinander agierenden Menschen voneinander nicht lassen. So stehen alle gegenüber allen in Verhältnissen der Anziehung und Abstoßung.

Der skizzierte Zwiespalt wirkt nicht nur nach außen und bestimmt die Beziehungen der Menschen untereinander, sondern zeigt sich auch in einer inneren Zerrissenheit jedes Einzelnen. Auf der einen Seite finden sich seine natürlichen selbstsüchtigen Begierden und Neigungen, die Rücksichtnahmen auf die Belange eines engen Kreises derjenigen, die er zu den Seinen rechnet, einschließen mögen. Hier folgt der Einzelne nur seiner natürlichen, Kant formuliert bisweilen, „wilde[n]“ Freiheit (ebd., 22). Auf der anderen Seite wünscht er sich aber „als vernünftiges Geschöpf“ im eigenen und im Gesamtinteresse ein Gesetz, „,welches der Freiheit Aller Schranken setzt“ (ebd., 23). Indem dieses Gesetz Ausdruck eines ,allgemeingültigen Willens“ ist, ist es Ausdruck der Freiheit eines jeden - freilich der nicht-natürlichen, kultivierten, gerechten Freiheit. Rousseau, der diesen Gedanken ein- und Kant zu Bewusstsein bringt, hält es für menschenunmöglich, beiden Seiten zugleich Genüge zu tun. Entweder ist man (natürlicher) Mensch (l'homme [naturel]) oder Bürger (citoyen) (Rousseau 1969, 248). Kant jedoch ist der Auffassung, dass genau dieser Antagonismus ausgehalten und produktiv gemacht werden kann und muss. Er allein erlaubt die Entwicklung aller Kräfte des Menschen als eines vernünftigen Tiers.

Nietzsche nimmt auf seine Weise an dem Diskurs teil. Er betont stets und emphatisch sein „Jasagen zu Gegensatz und Krieg“ (z.B. Nietzsche 1988f, 313). Mit den 
anderen, aber auch mit sich selbst soll der Mensch in Verhältnissen der Spannung stehen und Fertigkeiten „im Kriegführen“ ausbilden (Nietzsche 1988d, 121). Sein Verlangen mag umgekehrt dahin gehen, „dass der Krieg, der er ist, einmal ein Ende habe“ (ebd., 120). „Ausruhen“, „Ungestörtheit“, „Sattheit“ erscheinen ihm dann als Glück. Nur der schwächere Typus Mensch aber wird nach Nietzsches Überzeugung diese Lösung der Beruhigung anstreben. Der stärkere wird den „Lebensreiz und -Kitzel“ von Gegensatz und Krieg schätzen (ebd., 121).

In welchem Sinn aber tragen Krieg und Gegensatz nach Nietzsche zur Entwicklung und ,Erhöhung;, wie er sagt, des Menschen bei? Warum sind es nicht Frieden und Einvernehmen, in denen die besten Chancen zur menschlichen Entfaltung liegen? Auch sind die verschiedensten Gestalten von Krieg und Gegensatz denkbar. Welche Formen kennt und diskutiert Nietzsche? Welche sind anderen vorzuziehen und aus welchen Gründen? Auf welchen Triebkräften beruhen sie jeweils? Die Frage, ob es etwas gibt oder geben sollte, was Krieg und Gegensatz begrenzt, muss ebenfalls gestellt werden. Denn neben einer Vielheit von Kräften, die auseinanderstreben, haben wir es ja immer auch mit Einheiten zu tun, die als solche agieren. Individuen und Gesellschaften müssen als Einheiten identifizierbar und ansprechbar sein, auch wenn sie in sich komplex strukturiert sind.

Was Rousseau, Kant und andere veranlasst, über Diversität und Antagonismus der in Individuum und Gesellschaft wirkenden Kräfte nachzudenken, ist das Problem ihrer sinnvollen, vor allem aber gerechten Organisation. Kant sieht in der Arbeit an diesem Problem die Hauptaufgabe und wesentliche Herausforderung, mit der der Mensch konfrontiert ist. Angesichts seiner natürlichen Mitgift, die ihn in divergierende Richtungen treibt, hin- und hergerissen zwischen sich verschließendem Eigensinn und sich öffnendem Gemeinsinn, sei daher „die Rolle des Menschen sehr künstlich“ (Kant 1912, 23). Kennt auch Nietzsche eine Ethik des Konflikts?

Sicher liegt es nahe, in Nietzsches Philosophie des Willens zur Macht eine zentrale Klammer zu sehen, die seine Überlegungen zu allen soeben erwähnten Punkten zusammenhält. Meine Ausführungen zu den genannten Fragen gliedere ich wie folgt: (1) Agon versus Vernichtungskampf; (2) Schwacher versus starker Wille - Nietzsches Ethik des Konflikts.

\section{Agon versus Vernichtungskampf}

Ich beginne mit dem Typ von Konflikten, der in jüngster Zeit von den Lesern Nietzsches wohl am stärksten beachtet wurde, nicht zuletzt, weil er sich einer liberalen Auslegung fügt. ${ }^{1}$ Die Grundlinien agonaler Verhältnisse und des in ihnen liegen-

1 Vgl. hierzu die informative Darstellung, die James Pearson (2017, 16-18, 21-23 et passim) vorlegt. 
den produktiven Potentials stellt Nietzsche bekanntlich in dem frühen und von ihm selbst nicht veröffentlichten Stück „Homer’s Wettkampf“ vor.

Es ist nützlich, sich den Ausgangspunkt des kurzen, aber gewichtigen und wohlkomponierten Textes vor Augen zu führen. Nietzsche beginnt mit dem Begriff der ,Humanität‘. Wenn man ihn versteht als Ausdruck dessen, was uns von der Natur ,abscheidet “, wie Nietzsche schreibt, hätte man ihn missverstanden (Nietzsche 1988a, 783). Denn es gebe eine solche Abscheidung in Wirklichkeit gar nicht: „die ,natürlichen' Eigenschaften und die eigentlich ,menschlich' genannten sind untrennbar verwachsen“. Auch in seinen höchsten und edelsten Kräften sei der Mensch „ganz Natur“ und trage „ihren unheimlichen Doppelcharakter“ an sich (ebd.), will sagen: ein und dieselbe natürliche Kraft kann in diametral verschiedener Weise zum Ausdruck kommen und verliert diese Ambivalenz auch nicht. Nietzsche behandelt - exemplarisch - den Trieb zur Auseinandersetzung, zu Streit, Kampf und Krieg. Er behandelt ihn in seinen beiden Formen und damit in seinem „Doppelcharakter“, als Wettkampf oder Agon und als „Vernichtungskampf“ (ebd., 787). Von der Einleitung her ist bereits klar, dass stets beide Formen in der Welt und mit dem Menschen verbunden sein werden.

Wie in der „Geburt der Tragödie“ wendet sich Nietzsche, auch um im Kontrast zu modernen Vorstellungen und Vorurteilen genauer zu sehen, den Griechen und ihrem Verständnis von Kampf und Krieg zu. Sie gelten als „die humansten Menschen“; dennoch seien Züge der Grausamkeit und einer „tigerhaften Vernichtungslust“ an ihnen unverkennbar (ebd., 783). Nietzsche erwähnt in diesem Zusammenhang einige Details aus dem Umgang Alexanders mit besiegten Feinden. Wir schauten in „Abgründe des Hasses“; und die Griechen hätten es für eine Notwendigkeit gehalten, diesen Hass voll „ausströmen“ zu lassen, wie Nietzsche formuliert (ebd., 784). Im Gegensatz zur modernen Tendenz des Argwohns gegenüber dem Trieb zu Streit, Kampf und Krieg samt seinen Begleiterscheinungen wie dem Hass hebt Nietzsche hervor, dass die Griechen ihn weit eher akzeptierten.

In der Rede vom „Ausströmenlassen“ des Hasses klingt Nietzsches Beschäftigung

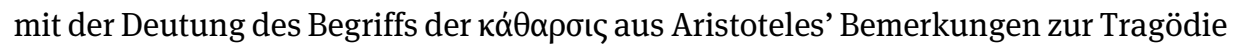
an (Aristoteles 1982, 18 f. = 1449b), die Jacob Bernays gegen Lessing vorgelegt hatte. Bernays hatte die „Reinigung der Affekte“, die Aristoteles zufolge die Tragödie durch Furcht ( $\varphi$ óßoৎ) und Mitleid ( $ع ̋ \lambda \varepsilon o \varsigma)$ bewirkt, in einem medizinischen Sinn als „,erleichternde Entladung“ dieser Affekte im Zuschauer aufgefasst. ${ }^{2}$ Lessing versteht die Reinigung der Affekte durch Furcht und Mitleid dagegen als deren „Verwan-dlung“ in „tugendhafte Fertigkeiten“, und das tragische Mitleid und die tragische Furcht

2 Vgl. Bernays 1970, 12. Die katharsis bei Aristoteles beschreibt er als „eine von Körperlichem auf Gemüthliches übertragene Bezeichnung für solche Behandlung eines Beklommenen, welche das ihn beklemmende Element nicht zu verwandlen oder zurückzudrängen sucht, sondern es aufregen, hervortreiben und dadurch Erleichterung des Beklommenen bewirken will.“ 
begreift er als genau solche Tugenden (Lessing 1967, 434 f.).Nietzsche bedient sich der Kategorie der ,Entladung، im Sinne der Interpretation von Bernays mit Blick auf menschliche Triebe, Kräfte, Emotionen und auch den Willen zur Macht immer wieder und bis in die spätesten Texte hinein. In „Homer's Wettkampf“ erklärt er, im zügellosen „Ausströmenlassen“ des Hasses „erleichterte sich“ den Griechen „die zusammengedrängte und geschwollene Empfindung“ (Nietzsche 1988a, 784) - und dies ist in einem nicht-moralischen, physiologisch-medizinischen Sinn gemeint.

Nietzsche merkt hier wie auch an anderen Stellen, beispielsweise später in der „Genealogie der Moral“, an, dass der moderne Mensch diese ganze kriegerische und vom Kampf geprägte griechische Welt kaum verstehe und „schaudern“ würde, wenn ihm dies je hinreichend gelänge (ebd.). Er notiert sodann den Übergang zu einer „edleren Kultur“ des Umgangs mit Krieg und Konflikt (ebd., 785). Der Trieb dazu, zu Krieg und Konflikt, ist stets und bleibend im Menschen angelegt, „furchtbar vorhanden“, stellt Nietzsche fest (ebd.). Wie sich ihm gegenüber verhalten?

Mit dieser Frage beginnt die Kultur, da sie den Trieb ins Bewusstsein rückt und so distanziert. Eine Weise, sich ihm gegenüber einzustellen, die Nietzsche erwähnt (ebd.), besteht darin, mit Lebensekel zu reagieren. Dies ist die Antwort des Pessimismus und der Lebensverneinung, die Nietzsche im Denken Schopenhauers und dessen Inspiration durch die Philosophie Indiens findet. In einigen Kulten der Griechen, zum Beispiel in der Orphik, sieht er ähnliche Tendenzen wirksam. Doch vermag die Antwort des Pessimismus in seinen Augen nicht zu überzeugen. Denn sie verweigert die Annahme der Herausforderung, welche die im Menschen angelegte Disposition zu Krieg und Gegensatz darstellt.

Im Mittelpunkt von Nietzsches Interesse steht dann, wie im Titel der kleinen Schrift angedeutet, eine andere Antwort der Griechen auf die Frage „was will ein Leben des Kampfes und des Sieges?“ (Nietzsche 1988a, 785). Es will den Gegensatz, den Antagonismus, als Stimulans zum Wettkampf, der die Entfaltung aller menschlichen Kräfte und Begabungen vorantreibt. Wie bei Kant ist die Selbstsucht des Individuums einschließlich der zugehörigen und zumeist negativ konnotierten Emotionen Eifersucht, Groll und Neid als Agens gedacht. Sie reizt zum Wettkampf; der Widerstand und vielleicht das Ärgernis, die der Andere bietet, werden zum Ansporn, es ihm gleichzutun oder ihn übertreffen zu wollen. Nietzsche zitiert bekanntlich Hesiod und bezieht sich auch auf entsprechende Belege bei Aristoteles. Zwietracht und Streit, für die die Göttin Eris stand, werden zum Guten gewendet: „Sie [Eris] treibt auch den ungeschickten Mann zur Arbeit; und schaut einer, der des Besitzthums ermangelt, auf den Anderen, der reich ist, so eilt er sich in gleicher Weise zu säen und zu pflanzen und das Haus wohl zu bestellen. [...] Auch der Töpfer grollt dem Töpfer und der Zimmermann dem Zimmermann, es neidet der Bettler den Bettler und der Sänger den Sänger."3

3 Nietzsche 1988a, 786. Vgl. Hesiod, Werke und Tage, 11-26 (2012, 82-85). 
Im Prinzip kann der Agon alle Lebensbereiche durchdringen. Und man kann dafür argumentieren, dass ein aktiv verfolgtes beständiges „Anstreben gegen Widerstehendes“ (Nietzsche 1988i, 52), dass der an keinem Sieg sich beruhigende „Durst nach Feinden und Widerständen und Triumphen“ (Nietzsche 1988e, 279) nach Nietzsches Dafürhalten das Fundament eines gelingenden Lebens bildet. Der Agon hält, so verstanden, die nötige Spannung aufrecht, ohne die es keine Entwicklung der Kräfte, die nur an Widerständen wachsen, und keinen Reichtum der Erfahrung, der sich nur im Durchgang durch Herausforderungen bildet, geben kann. Von einer wahrhaft so zu nennenden Lebenserfüllung könnte ohne dieses agonale Element dann nicht die Rede sein. Bernard Reginster spricht deshalb, in Anlehnung an Nietzsches Formulierung aus der späten Vorrede zur „Fröhlichen Wissenschaft“, von seiner Konzeption eines „neue[n]“, agonal verfassten „Glücks“.4

Weil der Agon schon einmal - nämlich im Griechentum - der „Lebensgrund“ gewesen sei, taugt die griechische Kultur in Nietzsches Einschätzung bis heute zum Vorbild (Nietzsche 1988a, 788). Interessant für uns, auch im Vergleich zum Kantischen Problemaufriss, mit dem wir begonnen haben, ist Folgendes: Nietzsche deutet an, dass die im Wettkampf fruchtbar werdenden, in der Selbstsucht des Einzelnen wurzelnden Gegensätze und Spannungen im griechischen Staat diese Produktivität auch einer gewissen Ordnung verdankten, durch die ebendiese Selbstsucht „gezügelt und umschränkt“ war (ebd., 790). Drei Elemente werden von Nietzsche genannt, und es wird zu prüfen sein, ob und wie sie sich in die moderne Welt übertragen lassen. Da ist zum einen die Identifikation mit dem Gemeinwesen. Im Wettkampf der Städte war es das Wohl der Vaterstadt, das dem Griechen vor Augen stand und das seinen Ehrgeiz nicht ins „Ungemessene und Unzumessende“ steigen ließ (ebd., 789). Dann ist es die Scheu vor den Göttern, mit denen er sich nicht messen darf, soll ihm solche Hybris nicht zum Verhängnis werden. Er wird an „das Vergängliche jedes Menschenlooses“ gemahnt (ebd., 787). Am wichtigsten jedoch ist ein Grundsatz, den Nietzsche mit dem eigentlichen Sinn des Ostrakismos, des Scherbengerichts, verbindet - und er zitiert ein Fragment des Heraklit, in dem dieser von den Ephesiern berichtet, die den Fähigsten unter ihnen zur Stadt hinaus jagten mit der Begründung: „Unter uns soll Niemand der Beste sein; ist Jemand es aber, so sei er anderswo und bei Anderen. “5 Es ist dies ein Prinzip, das der Suspendierung des Wettkampfs den Riegel vorschiebt.

In verschiedener Weise also scheint der antike Mensch bereit, Begrenzungen $\mathrm{zu}$ akzeptieren. Den modernen Menschen dagegen stellt Nietzsche als einen vor, dem dies nicht mehr selbstverständlich ist. Er sei „überall gekreuzt von der Unendlichkeit“, schreibt Nietzsche (1988a, 790). Das Problem der Integration und Desintegration diverser Kräfte zeichnet sich ab, das uns in dem Abschnitt über den starken und

4 Reginster 2007; vgl. dazu Nietzsche 1988c, 351.

5 Nietzsche 1988a, 788. Vgl. Heraklit, Frg. 121 (Diels-Kranz I, 1960, 178). 
schwachen Willen noch beschäftigen wird. Im Vergleich zu Nietzsches Lösung werden wir auch die von Rousseau und Kant ins Spiel gebrachte Idee eines ,allgemeingültigen Willens' betrachten, der sich in Recht und Gesetz ausdrückt. Der intensivste Antagonismus, der zwischen den einzelnen Gliedern einer Gesellschaft herrscht und sie lebendig hält, würde in diesem Modell nicht nach Gesichtspunkten von Schwäche und Stärke und auch nicht lokal, sondern im Blick auf den gemeinsamen Ausgangspunkt in der menschlichen Freiheit geordnet.

Genau solche universal gedachte Ansatzpunkte scheint Nietzsche im Auge zu haben, wenn er den modernen Menschen mit der besagten „Unendlichkeit“ fast belastet sieht und hinzufügt, dass „die Individuen im Alterthume freier [waren], weil ihre Ziele näher und greifbarer waren“ (ebd.). Gefeit gegen den Umschlag vom geordneten Agon in den Vernichtungskampf zeigen sich allerdings beide nicht. Den Gründen dafür wollen wir uns jetzt zuwenden.

Eine Hauptursache dafür, dass der Agon sich in den Vernichtungskampf verkehrt, sieht Nietzsche im Verstoß gegen den genannten wichtigsten Grundsatz des Wettkampfes, der seine Aussetzung verbietet. Kommt der Agon nämlich zum Stillstand, ist der Rückfall in die grauenhafte „Wildheit des Hasses und der Vernichtungslust“ (ebd., 791) Nietzsche zufolge wahrscheinlich. Dieses Phänomen, fährt er fort, zeige sich deutlich, wenn eine große Persönlichkeit durch eine „ungeheure glänzende That" nach seinem und seiner Mitbürger Urteil plötzlich dem Wettkampf entrückt, hors de concours sei (ebd.). Die Wirkung sei fast immer entsetzlich. Nietzsche führt Miltiades an, den Feldherrn der Schlacht bei Marathon, der einer früheren Kränkung wegen gegen die Bewohner der Insel Paros zu Felde zieht und sie verwüstet. Um sein Rachegelüst zu befriedigen, missbraucht er - nach dem Bericht Herodots ${ }^{6}-$ Ruf, Staatsvermögen und seine Bürgerehre, begeht einen Gottesfrevel und endet in Unehren. In ähnlicher Weise, fügt Nietzsche hinzu, führten die übermächtigen griechischen Staaten Athen und Sparta zuletzt durch Taten der Hybris ihren Untergang selbst herbei. Er kommt zu dem Schluss, „daß ohne Neid Eifersucht und wettkämpfenden Ehrgeiz der hellenische Staat wie der hellenische Mensch entartet. Er wird böse und grausam, er wird rachsüchtig und gottlos, kurz, er wird ,vorhomerisch““ (ebd., 792).

Das Fehlen geeigneter Gegenkräfte also raubt dem auf den Antagonismus hin angelegten Menschen ebenso wie dem Staat, in dem sich die Einzelnen in der Form eines politischen Gemeinwesens formieren, gleichsam das Betätigungsfeld. Die einseitig zugewachsenen Energien werden dann in einer nicht mehr fokussierten Weise, nämlich „ohne Neid Eifersucht und wettkämpfenden Ehrgeiz“ (ebd.), die stets Bestimmtes im Auge haben, gleichsam wahllos und entsprechend unangemessen entladen. Die Ordnungen brechen zusammen. Das Wohl der Vaterstadt, das nicht mehr verteidigt werden muss, verliert seinen Wert. Die Scheu vor den Göttern, die an das

6 Herodot, Historien, VI, 133-136 (1971, 287-293). 
„Vergängliche jedes Menschenlooses“ (ebd., 787) erinnerte, weicht der nicht mehr herausgeforderten Selbstschätzung und -überschätzung. Die Suspendierung des Wettkampfes, der stets auch die Grenzen der eigenen Leistungsfähigkeit offenbart, führt zum fälschlichen Glauben an die Allmacht und dadurch ins Verderben. Mit all diesen Überlegungen rückt jetzt die Frage nach dem Gewicht von Kräften und Gegenkräften, dem Verhältnis von Schwäche und Stärke in Individuum und Gesellschaft in den Blickpunkt.

\section{Schwacher versus starker Wille - Nietzsches Ethik des Konflikts}

Dass Nietzsche im Verlauf seines weiteren Nachdenkens über die Antagonismen, die zwischen Individuen und gesellschaftlichen Gruppen wirken, das „Princip des Gleichgewichts“ herausstellt (Nietzsche 1988b, 555) - und dies hat eingehend Volker Gerhardt gezeigt (Gerhardt 1983), ist nach dem bisher Erörterten verständlich. Das Prinzip meint das ausgeglichene Verhältnis zwischen zwei ungefähr gleich Mächtigen und bietet die ideale Voraussetzung auch für das Gelingen des Agons. Für keine der Seiten gibt es irgendeinen vernünftigen Grund, einander zu schädigen oder gar zu vernichten. Hier kann, ohne die produktive Spannung gegenseitiger Herausforderung aufzuheben, doch alles auf den gegenseitigen Nutzen, Vorteil und Gewinn abgestellt werden.

Mit ins Bild tritt jetzt außerdem ein Faktor, der bisher keine Rolle gespielt hat: die Diversität der Kräfte, Fähigkeiten und Leistungen der verschiedenen Individuen und Gruppen. So etwas wie ein arbeitsteiliges Szenario zeichnet sich ab und mit ihm nicht so sehr antagonistisch, sondern komplementär zueinander sich verhaltende Energien. Die Möglichkeit, sie fruchtbar $\mathrm{zu}$ machen, ergibt sich und wird von Nietzsche hervorgehoben, indem er die Bedeutung von Tausch und Austausch betont. „Jeder stellt den Andern zufrieden“, formuliert er in dem einschlägigen Aphorismus 92 aus dem ersten Band von „Menschliches, Allzumenschliches“, „,indem Jeder bekommt, was er mehr schätzt als der Andere. Man giebt Jedem, was er haben will als das nunmehr Seinige, und empfängt dagegen das Gewünschte“ (Nietzsche 1988b, 89). In solchem „Austausch unter der Voraussetzung einer ungefähr gleichen Machtstellung“ sieht Nietzsche bekanntlich jede Vorstellung von Gerechtigkeit wurzeln, den „anfängliche[n] Charakter der Gerechtigkeit“ (ebd.).

Freilich stellt sich sofort die Frage, wie der vielleicht sogar gewöhnlichere, gewiss aber ungleich interessantere Fall asymmetrischer Machtverhältnisse zu analysieren ist. Nietzsche diskutiert ihn unter dem Stichwort des ,starken“ bzw. ,schwachen Willens‘ und somit im Rahmen seiner Philosophie des Willens zur Macht. Das Problem, das die Tendenz zu Krieg und Gegensatz, also der Eigensinn der Menschen und Quell ihrer Produktivität wie auch ihrer Neigung zu Zerstörung und Selbst-zerstörung, 
aufgibt, wird natürlich erst dann wirklich sichtbar, wenn asymmetrische Machtverhältnisse in den Blick genommen werden. Die Gerechtigkeit als Austausch von Leistungen, Rechten und Wohlwollen zwischen gleich Mächtigen und sozusagen auf gleicher „Höhe“ - eine Metaphorik, die Nietzsche immer wieder einsetzt ${ }^{7}$ - ist ja wohlfeil und geschenkt. Entsprechend beschäftigt er sich sogleich im Anschluss an den erwähnten Aphorismus 92 aus „Menschliches, Allzumenschliches“, also im dreiundneunzigsten Stück, mit dem „Rechte des Schwächeren“ (Nietzsche 1988b, 90). Im Hintergrund beider Aphorismen steht die Darstellung des Gesprächs zwischen Athenern und Meliern über Macht und Recht in Thukydides' Geschichtswerk ${ }^{8}$, auf die Nietzsche explizit anspielt.

War - wie gesehen - bereits in der frühen Schrift über „Homer’s Wettkampf“ die Begrenzung des menschlichen Eigensinns oder der menschlichen Selbstsucht, wie Nietzsche sagt, zum Thema geworden, so lässt eine ausdrückliche Erörterung von Recht und Gerechtigkeit erwarten, dass jetzt die Legitimität einer solchen Ordnung zur Debatte steht. Wie verhält sich aber die Legitimität einer Ordnung des Konflikts zwischen Menschen zu ihrer jeweils unterschiedlichen Stärke oder Schwäche?

Dem genannten Aphorismus 93 aus „Menschliches, Allzumenschliches“ zufolge ist der Schwächere mehr oder weniger gezwungen, sich einem Mächtigeren zu unterwerfen. Freilich kann er auch aus dieser Position heraus noch „Bedingungen“ stellen (ebd.). Unter Anspielung auf Thukydides führt Nietzsche das Beispiel einer belagerten Stadt an. Sie kann den Wert und Nutzen, den sie als Stadt für den Mächtigen hat, wenn sie sich nicht vernichtet und verbrennt, sondern sich unbeschädigt der Herrschaft des Mächtigeren unterstellt, als Gewicht, als Verhandlungsmasse zur Geltung bringen. Auf diesem Grund können nach Nietzsche „Rechte festgesetzt werden“ (ebd.), die der Feind zugestehen mag, weil er seinen Vorteil an der Erhaltung der Stadt hat. Nietzsche kommt zu dem Schluss: „Insofern giebt es auch Rechte zwischen Sclaven und Herren, das heisst genau in dem Maasse, in welchem der Besitz des Sclaven seinem Herrn nützlich und wichtig ist. Das Recht geht urspünglich so weit, als Einer dem Andern werthvoll, wesentlich, unverlierbar, unbesiegbar und dergleichen ers cheint . In dieser Hinsicht hat auch der Schwächere noch Rechte, aber geringere“ (Nietzsche 1988b, 90 f.). Und dann fügt Nietzsche bekanntlich noch eine entsprechende Einschätzung von Spinoza an, die er bei Schopenhauer nachlesen konnte. ${ }^{9}$

Von der hier formulierten Position rückt Nietzsche, soweit ich sehe, im Grundsatz nicht mehr ab. Wie genau aber lautet seine Stellungnahme mit Blick auf das Problem, das uns hier beschäftigt: Was macht eine Ordnung des Konflikts zwischen Menschen

7 Vgl. die einschlägigen Aphorismen im „Neunte[n] Hauptstück“ von „Jenseits von Gut und Böse“ (Nietzsche 1988d, 205 ff.). Dazu Himmelmann 2017, 177-181, 188 (Anm. 15).

8 Thukydides, V, 84-116 (1966, 57-66).

9 ,[...] unusquisque tantum juris habet, quantum potentia valet (oder genauer: quantum potentia valere creditur)“ (Nietzsche 1988b, 91). Vgl. Spinoza 2010, 20; Schopenhauer 1986, 286 f. 
in ihrer individuellen, je unterschiedlichen Stärke oder Schwäche gerecht? Um klarer zu sehen, sollen einige Überlegungen des oben erwähnten Jean-Jacques Rousseau gleichsam als Kontrastfolie dienen. Nietzsche rechnete ja im übrigen Rousseau neben Epikur und Montaigne, Goethe und Spinoza, Plato, Pascal und Schopenhauer zu denjenigen, mit denen er sich stets „auseinandersetzen muss“ und von denen er sich „Recht und Unrecht geben lassen“ will (Nietzsche 1988b, 534).

In seinem „Contrat social“ nimmt Rousseau wie Nietzsche die Menschen in ihrer unterschiedlichen Stärke und Schwäche zum Ausgangspunkt seines Nachdenkens über die rechte Ordnung ihres Zusammenlebens. „Dabei werden die Menschen genommen, wie sie sind“ (en prenant les hommes tels qu'ils sont) (Rousseau 1964, 351). So spricht Rousseau wie Nietzsche von Herren und Sklaven oder genauer von denen, die sich für die einen oder anderen halten (ebd.). Die „Wirkungen“ (effets), die solche Verhältnisse von Stärke und Schwäche hervorbringen, sind für Rousseau evident: Der Schwache muss sich dem Starken beugen in dem Sinn, dass er ihm zu gehorchen gezwungen ist. Und solange er gehorcht, tut er nach Rousseau gut daran. Sobald er aber sein Joch abschütteln könne, tue er noch besser. Dann erlange er nämlich seine Freiheit durch dasselbe Recht wieder, das sie ihm geraubt hat (ebd., $351 \mathrm{f}$.).

Rousseau durchdenkt auch die Idee eines „Rechts des Stärkeren“ (le droit du plus fort). Stärke, erklärt er, sei ein „natürliches Vermögen“ (une puissance phisique). Nun sehe er überhaupt nicht, welche sittliche Verpflichtung (moralité) - die für Rousseau mit der Idee des Rechts verbunden ist - sich aus den Wirkungen der Stärke ergeben kann. Der Stärke zu weichen, sei ein Akt der Notwendigkeit, nicht des freien Willens (volonté), allenfalls ein Akt der Klugheit (ebd., 354).

Wenn man trotzdem einmal annehme, es gebe so etwas wie ein Recht der Stärke, so zeige sich schnell, meint Rousseau, die Absurdität des Gedankens. Sobald Stärke Recht schafft, ändere sich mit der Ursache immer auch die Wirkung. Denn jede Stärke, die eine andere übersteigt, folgt ihr dann im Schaffen von Recht nach. Weil der Stärkere also stets recht hat, handele es sich darum, es so einzurichten, dass man der Stärkere sei. Rousseau fragt: „Was ist das aber für ein Recht, das untergeht, wenn die Stärke endet?” (Qu'est-ce qu'un droit qui périt quand la force cesse?) (ebd.). Wird man gezwungen zu gehorchen oder lässt sich aus Klugheit darauf ein, ist es nicht mehr nötig, es aus einer rechtlichen Verpflichtung heraus zu tun. Und wenn man nicht mehr gezwungen oder aus Klugheit bewogen ist zu gehorchen, ist man dazu auch nicht mehr rechtlich verpflichtet. Man sieht also, schließt Rousseau, dass dieses Wort „Recht“ (ce mot de droit) der Stärke nichts hinzufügt (ebd.). Es besage hier überhaupt nichts.

Auf das Problem „Was macht eine Ordnung des Konflikts zwischen Menschen in ihrer individuellen, je unterschiedlichen Stärke oder Schwäche gerecht oder legitim?" scheint es im Rahmen des Ansatzes, den Nietzsche entwickelt, nur die Antwort zu geben: Eben die je unterschiedliche Stärke oder Schwäche der involvierten Menschen oder Gruppen. 
Genau diese Konsequenz seines Denkens zieht Nietzsche in einschlägigen Passagen der „Genealogie der Moral“ auch selbst. Nachdem er dort in einer subtilen Argumentation die neutralisierende und kultivierende Wirkung vorgeführt hatte, die die Einsetzung von Recht und Gesetz gegen blinde Rachegelüste und Ressentiments entfaltet (Nietzsche 1988e, $311 \mathrm{f}$.), kommt er zuletzt doch zu der wohlbekannten Schlussfolgerung: Rechtszustände dürften „vom höchsten biologischen Standpunkte aus“ betrachtet immer nur „Au sna h me-Zustände“ sein, die den „eigentlichen Lebenswillen" teilweise restringieren (ebd., 312 f.). Sie müssten sich deshalb dessen Gesamtzweck als „Einzelmittel“ unterordnen, als Mittel nämlich, „g rössere Macht-Einheiten zu schaffen“: „Eine Rechtsordnung souverain und allgemein gedacht [...] wäre ein lebensfeindliches Princip, eine Zerstörerin und Auflöserin des Menschen, ein Attentat auf die Zukunft des Menschen, ein Zeichen von Ermüdung, ein Schleichweg zum Nichts. -“ (ebd., 313). Es ist ebenso bezeichnend wie folgerichtig, dass Nietzsche über Ordnungen des Konflikts dann gar nicht mehr in Kategorien von Recht und Unrecht, Gerechtigkeit und Ungerechtigkeit spricht. Er arbeitet stattdessen mit den Kategorien von ,gut' und ,schlecht', die er auf Stärke bzw. Schwäche bezieht und gegen deren Pervertierung in das Gegensatzpaar ,gut' und ,böse‘ durch eine Ethik des Ressentiments er sich wendet (vgl. Himmelmann 2018). Es sind entsprechend Rangordnungen, gegliedert nach Graden von Stärke und Schwäche, die sich bilden und bilden sollen.

Jedes egalitäre Moment entfällt für Nietzsche. Bei Rousseau wie auch bei Kant war es dadurch gegeben, dass eine allen gemeinsame Freiheit (liberté commune) als Folge, wie Rousseau schreibt, der Natur des Menschen angenommen wird (Rousseau 1964, 352). Aus ihr leitet sich die Idee des Rechts ab. Nietzsche dagegen befürchtet bekanntlich, dass der Mensch zum antriebs- und spannungslosen „Heerdenthier“ verkommt (Nietzsche 1988d, 124-126; 145-147), sobald gewisse seiner Eigenschaften, Begabungen, Tugenden dem durchgängigen Wettkampf aller Kräfte entzogen und dann nicht mehr herausgefordert werden können. Im Grunde setzt Nietzsche - mit Rousseau gesprochen - allein auf den ,natürlichen Menschen' in seiner - wie Kant sagen würde - ,wilden Freiheit‘ und bricht mit der Idee des Bürgers. „Die Erreichung einer allgemein das Recht verwaltenden bürgerlichen Gesellschaft “ ist für Nietzsche sicher nicht wie noch für Kant „das größte Problem für die Menschengattung“ (vgl. Kant 1912, 22). Die „Gleichheit vor dem Gesetz“ wirkt für ihn vor allem nivellierend und inhibierend mit Blick auf die Lösung des Problems, das für ihn zählt: die Entfaltung des unabsehbaren Reichtums an Individualität, Eigenem und Abweichendem, der den ,großen“ oder ,höheren“ Menschen auszeichnet. Er soll nicht so vorgestellt werden, dass er dem Antagonismus der Kräfte entrückt wäre - dies war Nietzsche ja schon in dem frühen Text über „Homer’s Wettkampf“ klar. Er ist derjenige, der in sich die größten Gegensätze vereinigt und außer sich seine Grenze in der Stärke eines anderen Großen findet.

Was Nietzsche vorschwebt, ist eine nicht kodifizierte, situations- und fallbezogen sich vollziehende Selbstorganisation der Kräfte im Individuum und in der Gesell- 
schaft. „Die höchste Kraft, als Herrschaft über Gegensätze“ soll dabei „den Maaßstab [abgeben]“ (Nietzsche 1988h, 119). Die Einschränkung der Freiheit durch Recht und Gesetz braucht es dann nicht mehr; sie wäre sogar kontraproduktiv. Kants Sorge um den Menschen, der - ,aus krummem Holze“ - „seine Freiheit in Ansehung anderer Seinesgleichen [gewiß mißbraucht]“ und deshalb eines „Herrn “ bedarf, „der ihn nöthige, einem allgemeingültigen Willen, dabei jeder frei sein kann, zu gehorchen“ (Kant 1912, 23), teilt Nietzsche nicht. Er beklagt im Gegenteil ein Zuwenig an natürlicher, vom Egoismus geleiteter Energie:

\begin{abstract}
Bisher ist es der Mangel an feinem planmäßigen gedankenreichen Egoismus gewesen, was die Menschen im Ganzen auf einer so niedrigen Stufe erhält! Gleich heit gilt als verbindend und erstrebenswerth! Es spukt ein falscher Begriff von Eintracht und Frieden, als dem nützlichsten Zustande. In Wahrheit gehört überall ein starker A n t a g o n i s m us hinein, in Ehe Freundschaft Staat Staatenbund Körperschaft gelehrten Vereinen Religionen, damit etwas Rechtes wachse. Das Widerstreben ist die Form der Kraft - im Frieden wie im Kriege, folglich müssen verschiedene Kräfte und nicht gleiche dasein, denn diese würden sich das Gleichgewicht halten! (Nietzsche 1988g, 558).
\end{abstract}

Ob dieses Spiel der Kräfte, gegliedert nach Rangordnungen von Stärke und Schwäche, die sowohl von Klugheit, also agonal, als auch von Zerstörungswillen und Vernichtungslust geprägt sein können, nicht doch noch ein anderes, Stärke und Schwäche transzendierendes Maß verlangt, bleibt die Frage. Nietzsche steht in einem zwiespältigen Verhältnis zu der von Helmuth Plessner analysierten „Emanzipation der Macht“ (Plessner 1981). Er löst den Zusammenhang zwischen Macht und Recht auf, der für das Denken - wie Plessner erklärt - lange selbstverständlich war (ebd., 262). Den Gedanken des Rechts fasst Plessner als dasjenige, was die Macht zur „Verfügungsgewalt“ transformiert, die „Umschreibung verlangt und Definition braucht“ (ebd., 261). Jede solche „Festlegung“ schaffe Recht - unabhängig davon, ob es sich um Gewohnheitsrecht, geheiligte Sitte oder Recht durch Satzung und Gesetz handle. Rechtlich ausgeübte Macht unterscheidet sich damit grundlegend von Macht als bloßer Stärke, die sich ,im Vollzug“ zeige und im Vollzug die erreichte Position zu erkennen gebe. „Beim Menschen geht Macht also nicht in Mächtigkeit auf“, schließt Plessner, „sondern löst sich von ihr ab.“ Damit werde sie zu ,einer objektiv zu behandelnden Möglichkeit“ und figuriere „als Verfügungsgewalt von je besonderer Art im Rahmen einer Rechtsordnung, die ihrerseits auf sie angewiesen bleibt“ (ebd.). Diese Emanzipation vollzieht Nietzsche nicht mit; im Gegenteil, er widersetzt sich ihr. Für Plessner dagegen gehören Macht und Recht „strukturell zusammen“, ,auch wenn sehr oft das eine als Widerpart und Bedrohung des anderen erscheint, das Recht als Zügel und Schranke, die Macht als sprengende, anomische Kraft“ (ebd., 262). So unterstreicht Plessner auf seine Weise die von Kant geltend gemachte Ambivalenz, die sich in der ,geselligen Ungeselligkeit‘ des Menschen ausdrückt. Dieser These Kants hat Plessner im übrigen einen eindrucksvollen, kurzen und dichten Essay gewidmet (Plessner 1983). 
Nietzsche freilich treibt die Emanzipation der Macht in dem Sinn auf die Spitze, als es ihm um die „Freilegung des Begriffs der reinen Macht“ als einer „wertindifferenten Dynamik“ geht (Plessner 1981, 266), in der das menschliche Leben und Zusammenleben gründe und aus der so etwas wie Werte dann allererst entstehen - und vergehen. Auf seine Weise versucht sich Nietzsche an dem bereits von Rousseau erprobten Weg „von der Polis zur Natur“ (vgl. Spaemann 2008, 47-83). 


\section{Literaturverzeichnis}

Aristoteles, Poetik (1982): Poetik. Griechisch/Deutsch, Stuttgart.

Bernays, Jacob (1970): Grundzüge der verlorenen Abhandlung des Aristoteles über Wirkung der Tragödie (= Reprographischer Nachdruck der Ausgabe Breslau 1858), Hildesheim/New York.

Diels, Hermann/Kranz, Walther (Hg.) (1960): Die Fragmente der Vorsokratiker, 9. Aufl., Bd. 1, Berlin. Gerhardt, Volker (1983): Das ,Princip des Gleichgewichts‘, in: Nietzsche-Studien 12, 111-133.

Herodot (1971): Herodotus in Four Volumes, vol. III, Greek with an English translation by A.D. Godley, London/Cambridge.

Hesiod (2012): Theogonie. Werke und Tage, Griechisch - Deutsch, hg. von Albert von Schirnding, Berlin.

Himmelmann, Beatrix (2017): Nietzsche's Ethics of Power and the Ideas of Right, Justice, and Dignity, in: The Journal of Nietzsche-Studies 48/2, 171-189.

Himmelmann, Beatrix (2018): Luther, Nietzsche und das Problem des Bösen, in: Heit, Helmut/Sommer, Andreas-Urs (Hg.): 500 Jahre „Entrüstung der Einfalt“ - Nietzsche und die Reformation, Berlin/Boston (im Erscheinen).

Kant, Immanuel (1912): Idee zu einer allgemeinen Geschichte in weltbürgerlicher Absicht, in: Ders.: Gesammelte Schriften, hg. von der Preußischen Akademie der Wissenschaften, Bd. VIII, Berlin, 15-31.

Lessing, Gotthold Ephraim (1967): Hamburgische Dramaturgie, in: Lessings Werke, hg. von Kurt Wölfel, Bd. 2, Frankfurt a.M., 121-533.

Nietzsche, Friedrich (1988a): Homer's Wettkampf, in: Ders.: Kritische Studienausgabe, hg. von Giorgio Colli und Mazzino Montinari, Bd. 1, München/Berlin/New York, 783-792.

Nietzsche, Friedrich (1988b): Menschliches, Allzumenschliches, in: Ders., Kritische Studienausgabe, Bd. 2, München/Berlin/New York.

Nietzsche, Friedrich (1988c): Die fröhliche Wissenschaft, in: Ders.: Kritische Studienausgabe, Bd. 3, München/Berlin/New York, 343-651.

Nietzsche, Friedrich (1988d): Jenseits von Gut und Böse, in: Ders.: Kritische Studienausgabe, Bd. 5, München/Berlin/New York, 9-243.

Nietzsche, Friedrich (1988e): Zur Genealogie der Moral, in: Ders.: Kritische Studienausgabe, Bd. 5, München/Berlin/New York, 245-412.

Nietzsche, Friedrich (1988f): Ecce Homo, in: Ders.: Kritische Studienausgabe, Bd. 6, München/Berlin/New York, 255-374.

Nietzsche, Friedrich (1988g): Nachgelassene Fragmente: Nachlaß 1880-1882 (= Kritische Studienausgabe, Bd. 9), München/Berlin/New York.

Nietzsche, Friedrich (1988h): Nachgelassene Fragmente: Nachlaß 1884-1885 (= Kritische Studienausgabe, Bd. 11), München/Berlin/New York.

Nietzsche, Friedrich (1988i): Nachgelassene Fragmente: Nachlaß 1887-1889 (= Kritische Studienausgabe, Bd. 13), München/Berlin/New York.

Pearson, James S. (2017): Nietzsche's Philosophy of Conflict and the Logic of Organisational Struggle (Doctoral Thesis, Manuscript), Leiden.

Plessner, Helmuth (1981): Die Emanzipation der Macht, in: Gesammelte Schriften, Bd. V, Frankfurt a.M., 259-282.

Plessner, Helmuth (1983): Ungesellige Geselligkeit. Anmerkungen zu einem Kantischen Begriff, in: Gesammelte Schriften, Bd. VIII, Frankfurt a.M., 294-306.

Reginster, Bernard (2007): Nietzsche's ,New Happiness. ' On Longing, Boredom, and the Elusiveness of Fulfillment”, in: Philosophic Exchange, vol. 37, 17-40. 
Rousseau, Jean-Jacques (1964): Du contrat social, in: Ders.: Euvres complètes, t. III, Paris, 347470.

Rousseau, Jean-Jacques (1969): Émile ou de l'éducation, in: Ders.: đuuvres complètes, t. IV, Paris, 239-868.

Schopenhauer, Arthur (1986): Parerga und Paralipomena II, in: Ders.: Sämtliche Werke, hg. von Wolfgang Frh. von Löhneysen, Bd. V, Frankfurt a.M.

Spaemann, Robert (2008): Rousseau - Mensch oder Bürger. Das Dilemma der Moderne, Stuttgart. Spinoza, Baruch de (2010): Politischer Traktat/Tractatus politicus, Lateinisch-Deutsch, neu übersetzt und herausgegeben von Wolfgang Bartuschat, Hamburg.

Thukydides (1966): Thucydidis Historiae, vol. II, ed. Henry Stuart Jones und Johannes Enoch Powell, Oxford. 